\title{
THE EVOLUTION OF CLOSE BINARIES AND THE FORMATION OF THE WOLF-RAYET STARS
}

\author{
BOHDAN PACZYŃSKI \\ (Institute of Astronomy, Polish Academy of Sciences, Warsaw, Poland)
}

I shall discuss here the evolution of massive binaries in the case $B$. In this case the initially more massive component fills up the Roche lobe after the exhaustion of hydrogen in the core. By a massive binary I understand the binary in which the phase of a rapid mass exchange is terminated by helium ignition in the core of the initially more massive star. The helium ignition stops the contraction of the core and the expansion of the envelope of that component. In a small mass star the degeneracy plays the role of helium ignition in this respect. The last case will be discussed by Dr. A. Weigert in his lecture.

The evolution of the primary component of $9 M_{\odot}$ in the case $B$ was computed by Kippenhahn and Weigert (1967) and is shown in Figure 1. The first part of the evolu-

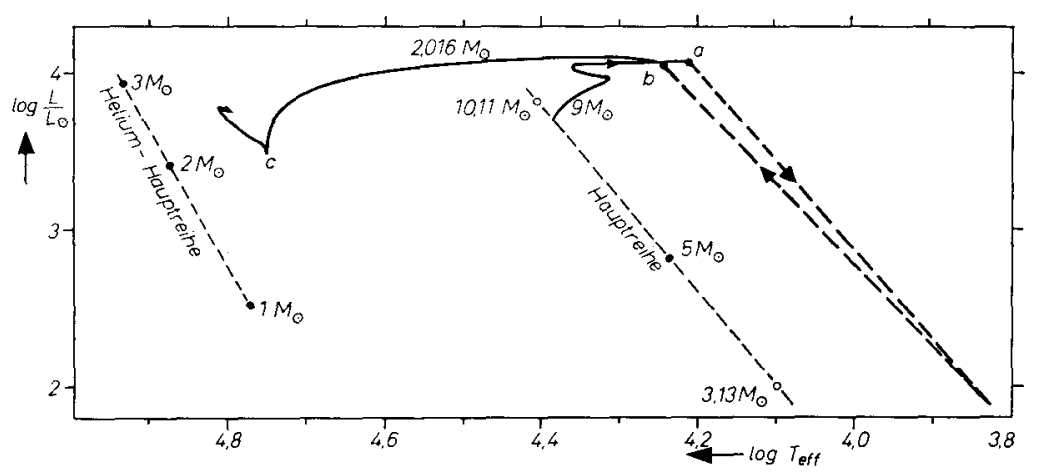

FIG. 1. Evolutionary track on the theoretical $H-R$ diagram for the primary component of the close binary studied by Kippenhahn and Weigert (1967, case B).

tionary track is the same as for a single star. The mass exchange begins after the Roche lobe has been filled up by the primary. At the beginning the mass transfer takes place on the thermal time-scale of the envelope of this star and is therefore extremely rapid. When the larger part of the hydrogen-rich envelope has been lost the mass exchange slows down and proceeds on the thermal time-scale of the contracting helium core. The process is terminated by the helium ignition. Later on the star evolves on the local nuclear time-scale of the hydrogen-burning shell source that causes the decrease of

Perek (ed.), Highlights of Astronomy, 409-413. (O I.A.U. 
stellar radius. The original primary approaches the sequence of homogeneous heliumburning models on the H-R diagram.

Similar computations were carried out by Paczyński $(1967 b)$ for a star of $16 M_{\odot}$. The evolution was followed through the phase of the most rapid mass exchange, and the results were in agreement with those obtained by Kippenhahn and Weigert. From the Weigert's discussion of the sequences of inhomogeneous models for helium-burning stars (Giannone, 1967) it follows that the evolution of massive binaries in case $B$ should practically always look like that shown in Figure 1. This means that as a result of mass exchange we get a detached binary with the initially more massive component resembling closely pure helium star. The other component is now more massive and close to the zero-age main sequence. It was suggested by Paczyński $(1967 a, b)$ that we have here a system with the Wolf-Rayet type component.

We shall summarize here some basic observational data concerned with the $\mathrm{W}-\mathrm{R}$ phenomenon. These stars have spectra with very prominent and wide emission lines of ionized helium and highly ionized nitrogen or carbon. The surface temperatures are very high, probably of the order of $50000^{\circ} \mathrm{K}$ (Kuhi, 1966). The average visual absolute magnitude is near -4 . The bolometric corrections are certainly large but unknown. Many W-R stars show small intrinsic-light variations. About $50 \%$ of the $\mathrm{W}-\mathrm{R}$ stars are known to be members of the binary system with the other component being a normal OB star. The photometric observations of the eclipsing systems of this type suggest that the surface brightness of the W-R star is higher than that of the OB component (Kron and Gordon, 1950). The basic data for eleven Wolf-Rayet binaries with known orbital periods are given in Table 1. Five of them are eclipsing variables. We see that the typical mass of the $\mathrm{W}-\mathrm{R}$ component is slightly below $10 M_{\odot}$, that of the $\mathrm{OB}$ star near $25 M_{\odot}$, and the average mass ratio $M_{\mathrm{WR}} / M_{\mathrm{OB}}=0 \cdot 3$.

We think that the high luminosities and surface temperatures of the $\mathrm{W}-\mathrm{R}$ stars are not compatible with our knowledge of the position occupied on the $\mathrm{H}-\mathrm{R}$ diagram by the massive stars in the phase of gravitational contraction towards the main sequence (Iben, 1965). It seems that the hypothesis that those stars are in advanced stage of stellar evolution (Burbidge and Burbidge, 1958; Limber, 1964; Divine, 1965), presumably in the helium-burning phase, and that they have lost in some way almost the totality of the hydrogen-rich envelope may account much better for their luminosities and surface temperatures, and perhaps for some anomalies in the abundances of the elements like hydrogen, carbon and nitrogen. The evolution of a massive binary in case $B$ may account in the most natural way for most of the properties of those $\mathrm{W}-\mathrm{R}$ stars that are members of binary systems. The masses, mass ratios and the periods of the observed binaries (Table 1) fall into the range of these quantities derived from the theoretical considerations (Paczyński, 1967b). The hypothetical model binaries with the initial masses of the primary in the range of 15-30 $M_{\odot}$ appear to be the best candidates for turning into the known $\mathrm{W}-\mathrm{R}$ binaries.

It is well known that the W-R stars split into WC and WN sequences. It is not clear 

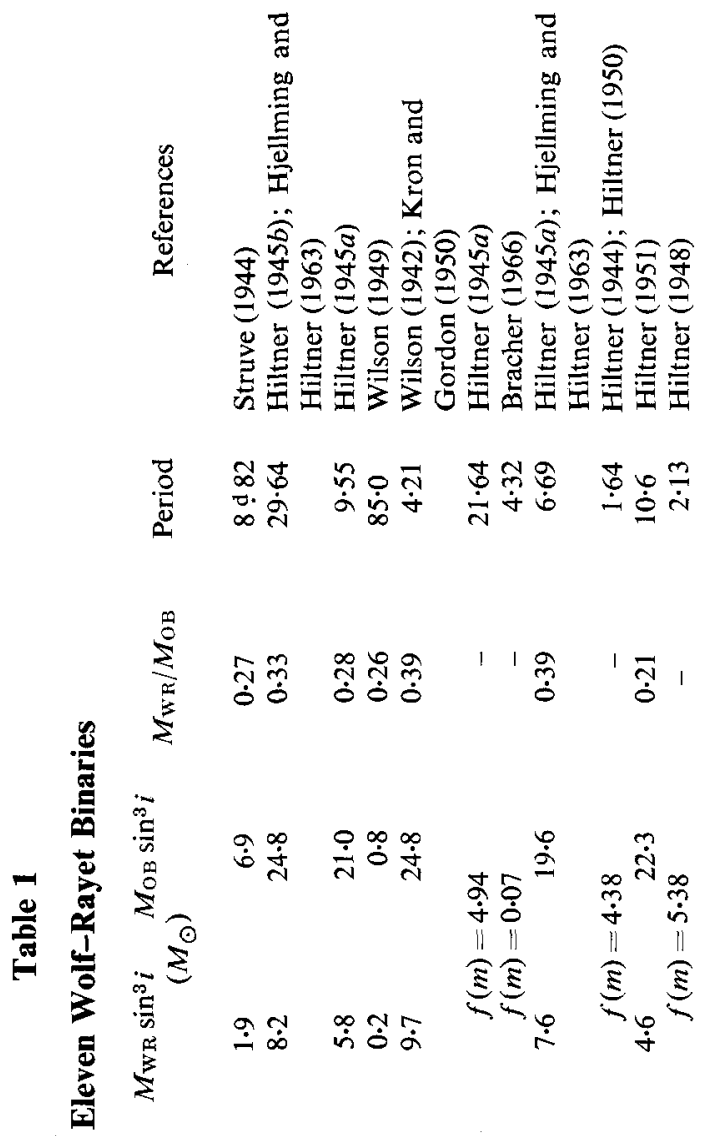

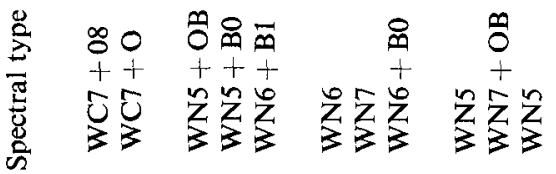

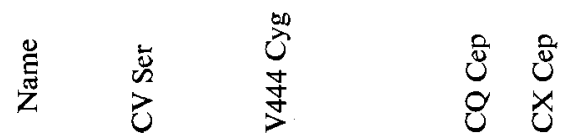

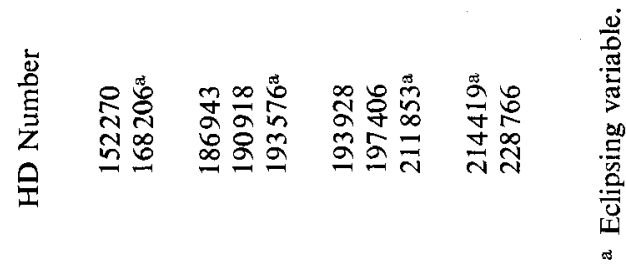


whether this is the result of different abundances or of different excitation conditions in the atmospheres. We should like to show that genuine abundance differences are to be expected if our model is correct. In the inner half of the original mass of the primary component almost all carbon and substantial fraction of the oxygen are transformed into nitrogen in the $\mathrm{CNO}$ cycle during the phase of core hydrogen burning. It is very likely that after the mass exchange the $\mathrm{W}-\mathrm{R}$ component will have a nitrogen-rich and carbon-poor atmosphere. Now, as a result of $\mathrm{N}^{14}(\alpha, \gamma) \mathrm{F}^{18}$ $\left(\beta^{+} v\right) \mathrm{O}^{18}$ reactions all nitrogen in the core will be transformed into oxygen, and carbon content will increase following helium burning. In massive helium stars the convective cores are large and have their masses almost constant during the evolution. We may expect that a large change in the $\mathrm{C} / \mathrm{N}$ ratio will be present at the boundary of the convective core. If the star has a mass above $7-8 M_{\odot}$ it will be probably vibrationally unstable and will eventually eject all the excessive mass (Boury and Ledoux, 1965). The convective core has a mass of $8 M_{\odot}$ for a helium star of $12 M_{\odot}$ (Divine, 1965). Therefore, if the mass of our star after the mass exchange exceeds $12 M_{\odot}$ it may, as a result of further mass loss, uncover those layers that were once within the convective helium-burning core. In that case the atmosphere will have no nitrogen and hydrogen, but carbon and oxygen may be abundant.

It is not clear whether in all massive binaries following the evolution corresponding to the case $B$ the initially more massive component may, after the mass exchange, show the W-R type characteristics. If not, the duplicity of the corresponding stars might be difficult to discover, as the helium component may not be bright enough in the visible light. This may be expected in the case of the binary studied by Kippenhahn and Weigert (1967). It is possible that vibrational instability of the helium star is required to generate W-R type spectrum. This instability, if present, might lead to light variations with a period of $0_{0}^{d} 01-0$. 04 . It would be interesting to see whether this can be observed. It would be also very interesting to know whether there are any single $\mathrm{W}-\mathrm{R}$ objects that belong to the extreme population I, and how much they are different from the $\mathrm{W}-\mathrm{R}$ binaries.

\section{References}

Boury, A., Ledoux, P. (1965) Ann. Ap., 28, 353.

Bracher, K. (1966) Astr. J., 71, 156.

Burbidge, E. M., Burbidge, G. (1958) in Encyclopedia of Physics, Vol. 51, Springer-Verlag, BerlinGöttingen-Heidelberg, p. 134.

Divine, N. (1965) Astrophys. J., 142, 824.

Giannone, P. (1967) Z. Astrophys. 65, 226.

Hiltner, W.A. (1944) Astrophys. J., 99, 274.

Hiltner, W.A. (1945a) Astrophys. J., 101, 356.

Hiltner, W.A. (1945b) Astrophys. J., 102, 492.

Hiltner, W.A. (1948) Astrophys. J., 108, 56.

Hiltner, W.A. (1950) Astrophys. J., 112, 477.

Hiltner, W.A. (1951) Astrophys. J., 113, 317.

Hjellming, R. M., Hiltner, W.A. (1963) Astrophys. J., 137, 1080. 
Iben, I. Jr. (1965) Astrophys. J., 141, 993.

Kippenhahn, R., Weigert, A. (1967) Z. Astrophys., 65, 251.

Kron, G. E., Gordon, K.C. (1950) Astrophys. J., 111, 454.

Kuhi, L.V. (1966) Astrophys. J., 143, 753.

Limber, D.N. (1964) Astrophys. J., 139, 1251.

Paczyński, B. (1967a) I.A.U. Colloquium "On the Evolution of Double Stars", Observatoire

Royal de Belgique, Communications, Série B, No 17, 111.

Paczyński, B. (1967b) Acta Astr., 17, 355.

Struve, O. (1944) Astrophys. J., 100, 384.

Wilson, O.C. (1942) Astrophys. J., 95, 402.

Wilson, O.C. (1949) Astrophys. J., 109, 76.

\section{DISCUSSION}

Underhill: In considering Wolf-Rayet stars it is important to realize

(i) that they are really very constant stars - light and spectrum variations are the exception, not the rule;

(ii) a great variety of objects is included in the class Wolf-Rayet including central stars of planetary nebulae; thus one model need not explain all objects;

(iii) the evidence concerning the surface temperatures is very weak - we can only say that these temperatures are about the same as those of $O$ stars;

(iv) our theories of spectrum-line formation are too rudimentary to permit any deductions about abundances.

Paczynski: What do you think about estimates of the surface temperature from the depths of eclipses?

Koch: It is not possible to infer $\left(J_{\mathrm{WR}} / J_{\mathrm{OB}}\right)$ from the ratios of the eclipse depths in those eclipsing binaries containing WR stars unless bandpass effects are allowed for.

Mammano: A WR nucleus of a planetary nebula has been recognised by F. Bertola (Publ, astr. Soc. Pacific, 76, 1964, 241) as being of type WN. Can this be taken into account in your scheme?

Paczynski: I know that some nuclei of planetary nebulae show $\mathrm{W}-\mathrm{R}$ type spectrum. But I do not think that these objects belong to the extreme Population I. 